\title{
A multifaceted semantic web service description of the pervasive information system
}

\author{
Fatma Achour, Anis Jedidi, Faiez Gargouri \\ MIRACL \\ Multimedia, Information Systems and Advanced Computing Laboratory \\ Sfax University, Tunisia \\ fatma.achour@gmail.com \\ anis.jedidi@isimsf.rnu.tn \\ faiez.gargouri@isimsf.rnu.tn
}

\begin{abstract}
The technique of adapting the web service allows the user to establish reusable, interoperable, flexible and context-aware applications. In fact, the pervasive system specification is provided by the context-aware applications since it is dynamically adapted. Furthermore, we seek to offer a dynamic description of the web services. This objective, however, can be achieved by adding contextual information structures to the web service description. In the literature, more research works strive to combine the contextual description with the web service description using the OWL-S structure.
\end{abstract}

In fact, the main objective of this paper is to propose structures with two levels for the contextual description of the pervasive system. This description is based on the generic model for the pervasive system design. We, also, suggest integrating such a description into the OWL-S structure.

\section{Indexing terms/Keywords}

Adaptation, Pervasive system, Ontology, OWL, OWL-S, Design.

\section{Academic Discipline And Sub-Disciplines}

Science computer

\section{SUBJECT CLASSIFICATION}

Science computer Subject Classification

\section{TYPE (METHOD/APPROACH)}

Pervasive computer, mobile system, semantic web service, ontology.

\section{Council for Innovative Research}

Peer Review Research Publishing System

\section{Journal: INTERNATIONAL JOURNAL OF COMPUTERS \& TECHNOLOGY}

Vol 8, No 2

editor@cirworld.com

www.cirworld.com, member.cirworld.com 


\section{INTRODUCTION}

The rapid evolution of new technologies steps up the complexity of the user's mobile environment. This is due to, to a great extent, the expansion of the various available services, the developments in sensor and device technologies leading to potentially large numbers of sensors and devices in the user's environment as well as the increasing number of heterogeneous communication networks. The principal objective of pervasive computing is to provide an intelligent environment so that the user can manage this situation with the minimum intervention. This goal, actually, can be achieved by dealing with the services and the resources in a dynamic way that may best meet the user's needs and preferences.

In this, adaptability is deemed to be a key aspect in the pervasive computing systems. Therefore, the system needs to adapt its functionality and behavior depending on the context of the user and the resources that are available at any instant. Although many research works highlight the idea of implementing and establishing adaptation in pervasive systems, none of them is interested in the design of adaptation in pervasive systems. We attempt, in this work, to suggest a system that can be conceptually adapted to the pervasive system. This system is based on semantic web services. Therefore, we have proposed a classification of contextual information for pervasive systems and used the OWL-S structure to integrate such information into the web services. In fact, the OWL ontology is a solution to support greater automation in the service selection and invocation and the automated translation of message content between the heterogeneous interoperating services and the service composition.

The pervasive system is described by contextual information. In this paper, we propose the contextual information classification and the architecture based on the web services for the pervasive system adaptation. This is the reason why we offer OWL-S structures to describe the web services based on an existing generic model to design the pervasive systems.

This paper is organized as follows. First, we study some concepts related to our research domain such as the pervasive system and the semantic web services. Second, we present the existing OWL-S extension to describe the semantic web services. Finally, we present our purpose that concerns the proposal of contextual description of the pervasive system.

\section{THE PERVASIVE SYSTEMS}

The advances of the intelligent-system and the need for transparency in the user's interaction with digital services have upgraded the notion of pervasive computing. The pervasive or ubiquitous computing was first introduced by Weiser as his vision of the computing future in the 21 st century

Weiser [Weiser, 1991] believed that the exponential evolution of data, software, hardware and connectivity would generate new environments rich of computing elements that lack proper interaction. As a result, he presented a paradigm where the computing elements would disappear from the user's consciousness while functioning homogeneously in the background of his environment. The final objective is to provide the user with omnipresent and seamless services available whenever and wherever they are needed [Park et al., 2004].

When the digital services kept increasing, many applications appeared. These applications can sometimes replace human beings. Thus, technology becomes a principal factor that could affect and improve life quality and business productivity by ensuring higher interoperability between the different business partners and the surrounding dynamic environments. With all these dynamic elements, a growing demand for easier, mobile, transparent and seamless interaction is sought in order to adapt it to the user's situations, abilities and needs.

\section{THE SEMANTIC WEB SERVICE DESCRIPTION}

The convergence of the semantic Web with the service oriented computing is manifested by the Semantic Web Services technology. In fact, it addresses the major challenge of automated, interoperable and meaningful coordination of web services to be carried out by intelligent software agents.

Additionally, each semantic service description framework can be characterized with respect to (1) what kind of service semantics are described, (2) in what language or formalism, (3) allowing for what kind of reasoning upon the abstract service descriptions.

In this paper, the major goal is to adapt the semantic web services to the different functionalities of the pervasive system. Essentially, the emphasis is laid on the contextual description of the different functionalities and the integration of this contextual information in the semantic web service description. Accordingly, the existing OWL-S structure is exploited in order to extend this structure.

\section{The OWL-S Ontology}

OWL-S [3] is a Web Services ontology that specifies a conceptual framework to describe the semantic web services. OWL-S is also a language based on the DARPA work of its DAML program and takes the result of DAML-S (DARPA Agent Markup Language Service). It was incorporated into W3C in 2004, within the interest group of semantic web services at the OWL recommendation [4]. The original purpose of OWL-S is to implement semantic web services. OWL-S is based on OWL to define the abstract categories of entities, events... in terms of classes and properties. OWL-S uses this ontology language description to define a particular ontology for the web services. This ontology is used to describe the web service properties as well as its services available to the public. The OWL-S structure regroups a set of ontology. 
Each one provides a functionality to describe the web service semantically. The ontology main classes described by OWL$S$ are defined as follows [3];

- The ontology "Service": represents the main class of OWL-S. It covers the web service general properties. This class has a"Service Profile" described by a "Service Model" and supports a "Service Grounding".

- The ontology "ServiceProfile": explains the functionality of the service and what it requires of the other agents. It also describes the service in terms of what it does to allow a requester to see if the proposed service suits him.

- The ontology "ServiceModel": defines the functioning of the web service and serves to explain how the service works. A web service can be seen as a process. For this reason, the "ServiceModel" has a subclass called the "ProcessModel".

- The ontology "ServiceModel": provides the information necessary for the use of the web service. In fact, you should choose the appropriate protocol to access the service, the message format, the way to serialize, the used transport mechanisms and the way the addressing mode is utilized.

- The ontology "Resources": provides information about the resources used by the web service. Web services often need resources to run. These resources are many and various. It is, therefore, vital to regroup them in the ontology.

The necessity to use the OWL-S ontology is justified by the creation of a semantic web service that has a dynamic description. This dynamic is provided by the addition of contextual descriptions to the OWL-S structure. The description depends on the use of the context of a pervasive system.

\section{The existing OWL-S extension to describe the semantic web services}

In the literature concerned with the web service adaptation, there are several works that take the advantage of the existing OWL-S structure to describe the different contexts. In this paper, we discuss two research works of Qiu et al., [8], [7] and Ben Mokhtar [5]. Qiu et al., research works propose an adaptation system based on the service composition approach. To do this, the authors offer three context categories [8].

- The user context ("U-Context"): this category specifies the context information about the user. In this context, the authors defined two types of contextual information: the user static context (profile, interest, and preferences) and the user dynamic context (location, current activity and task trying to achieve).

- The web service context ("W-Context"): this category includes the not-functional contextual information (price, execution time, confidence degree)

- The environmental context ("E-Context"): this category collects the context information about the user's environment (time, date, etc.).

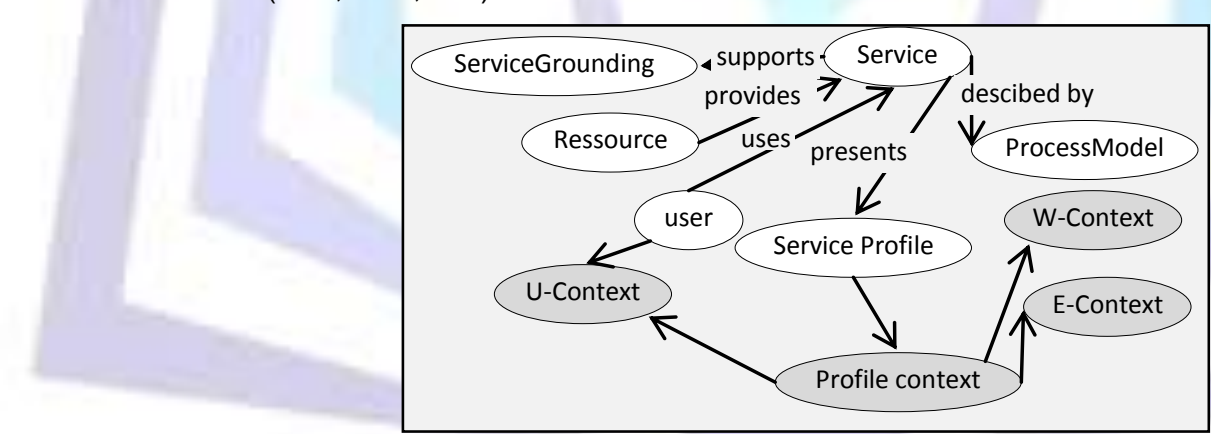

Fig. 1: The OWL-SC Ontology [7].

Each context category is represented by the OWL ontology and is integrated in the existing OWL-S extension ontology to introduce the OWL-SC (OWL-S for context) [7]. The latter is intended to describe a general contextual information (see Figure 2) based on the user's description.

The proposed structure focuses only on the user context description. However, it presents a vision for the integration or the addition of more information to the OWL-S structure.

Ben Mokhtar et al., research works propose a system to adapt the web services to a pervasive environment [5]. The context definition includes the description of four types of contextual information: the context sensors, services, devices and users. In addition, the contextual adaptation in this work is based on:

- The service representation: the authors describe the services using OWL-S extended with context information. This information is decomposed into a high level context attributes, preconditions and contextual effects.

- The user task representation: the user task representation is performed while extending the OWL-S service model ontology. To do this, the authors propose to integrate the quality conditions service descriptions and the context conditions required by the user task in the OWL-S structure. 


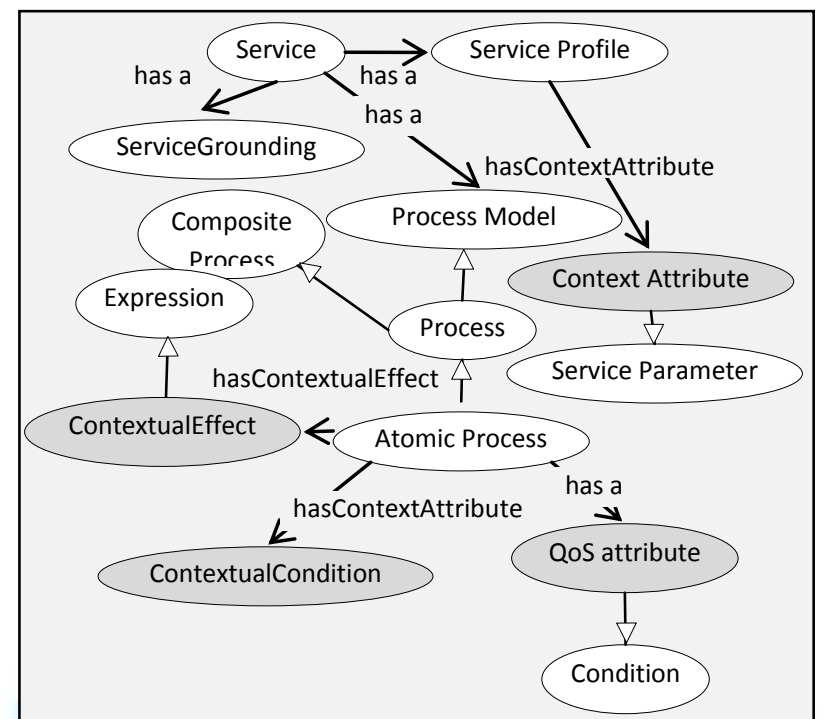

Fig. 2: The OWL-S ontology extension for the pervasive system [5]

The context information description is performed by means of an OWL extension ontology, the adaptation is carried out by applying a finite-state automaton.

Ben Mokhtar et al., their proposal concerns the contextual information integrated in the OWL-S structure, but this information is not related to the pervasive system description. We focus on, via this work, the integration of pervasive contextual information in the OWL-S structure.

\section{THE GENERAL MODEL USED TO DESCRIBE CONTEXTS}

\section{The context definition}

The most widely referenced definition of context is given by Dey et al [1] and states that the context is "Any information that can be used to characterize the situation of an entity. An entity is a user, a place, or a physical or a computational object that is considered relevant to the interaction between a user and an application, including the user and the application themselves". Using Dey's definition and our conception about context in relation to its describers, we consider the term context as an operational term whose definition depends on the interpretation of the operations involved in an entity at a particular time and space rather than the inherent characteristics of the entity.

\section{The general model used to describe the proposed semantic web service}

The description of the proposed web services is based on a general model [2]. The latter is a UML class diagram representing the intersection result of four existing models to describe the pervasive systems: SOUPA, Activity, and CSCP COMANTO. The classes defined in this diagram (see Figure 3 ) are described below:

- Agent: this class is used for the presentation of different actors in a pervasive system. It assembles the human actor and device actor.

- Person: presents all human actors.

- Device: presents the peripheral devices in a pervasive system.

- Service: presents the services offered by each device.

- Network: regroups the characteristics of different types of network.

- Location coordinate: represents the spatial relation between the different locations in a pervasive system.

- Preference: presents the information profile of a person who realizes the activity in a pervasive system.

- Activity: presents the characteristics of the activity requested by the user.

- Rules: regroups the different rules of activity, person and network interacting in a pervasive system.

- Time: presents the characteristics of a temporal thing and the relation of different things in a pervasive system.

- Location: represents the characteristics of localizations of human and mobile devices in a pervasive system.

- Role: represents the role of the users in the pervasive system. 


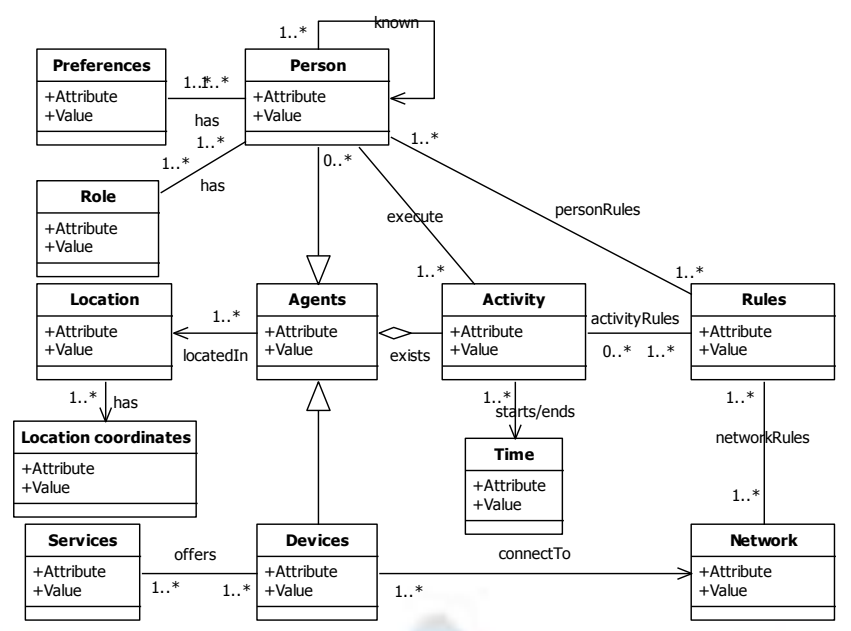

Fig. 3: Generic model used for the description [2].

After the model analysis, we found that the pervasive system contains the description of six contextual information categories:

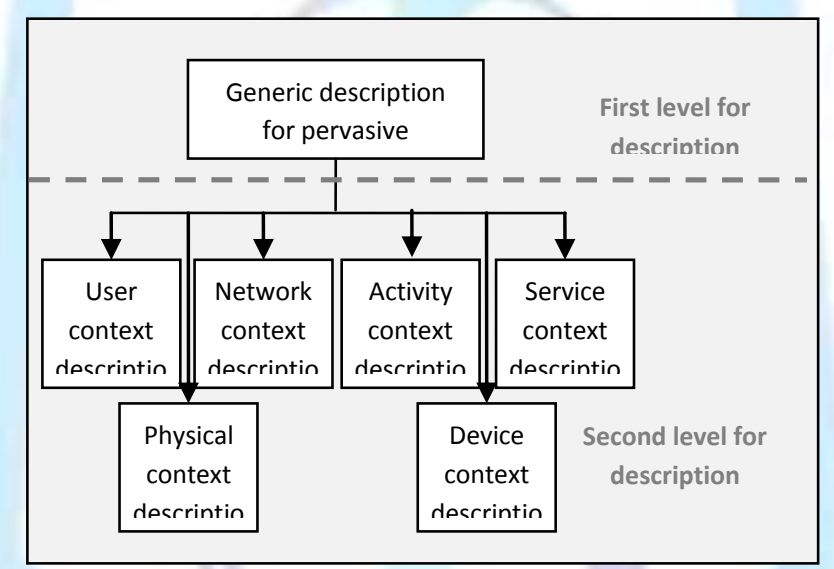

Fig. 4: The two levels of description

- User Context (Who?): contains information describing the human user situation, either directly or indirectly. The classes that represent this context type: person", "preferences", "role" and "rules".

- Physical Context (Where?): represents the physical environment in a pervasive information system. The classes that represent this type of context: "location" and "location coordinates".

- Network context (where?): combines the characteristics of all available networks in pervasive information systems. The classes that represent this type of context: "network" and "rules."

- Activity context (What happens, when?): combines information that represents the activity and the execution time of this activity in a pervasive information system. The classes that represent this type of context: "activity", "time" and "rules".

- Device context (What can be used?): represents the profile and device activity in a pervasive information system. The classes that represent this type of context are: "activity", "time" and "rules."

- Service context (What can be achieved?): regroups information about the functions that the pervasive system can provide. The classes that represent this type of context, "services" and "rules."

\section{PROPOSAL: TWO-LEVEL DESCRIPTION TO SELECT A WEB SERVICE}

The objective of this work is to propose a description for semantic web services. This description is composed of two levels. The first one presents a generic pervasive system description. But the second one is more detailed as it includes the ontology involving the pervasive system low-level description.

\section{The first level of the web service description}

The first level is represented by a semantic web service that generally describes the pervasive environment. It has all the necessary information about each web service context shown in the second level. This information is described in an 
extended OWL-S ontology (see Figure 5). The extended OWL-S ontology includes the basic information examining a pervasive system.

The pervasive context is presented by the "PervasiveContext" OWL class. The activities in a pervasive system are presented by the "A-Context" OWL class. They exist in a device. The latter is symbolized by the "D-Context" OWL class. Each "D-Context» device offers services in a pervasive system.

The services are presented by the "S-Context" OWL class. The latter regroups the characteristics of the services provided by the pervasive system. All the devices existing in a pervasive system are interconnected via multiple networks. These networks are modeled through the "N-Context" OWL class. The two classes "D-Context" and "U-Context" represent the entire agent that exists in a pervasive system. For this reason, we placed the two classes as a sub-class of the "Agent" OWL class.

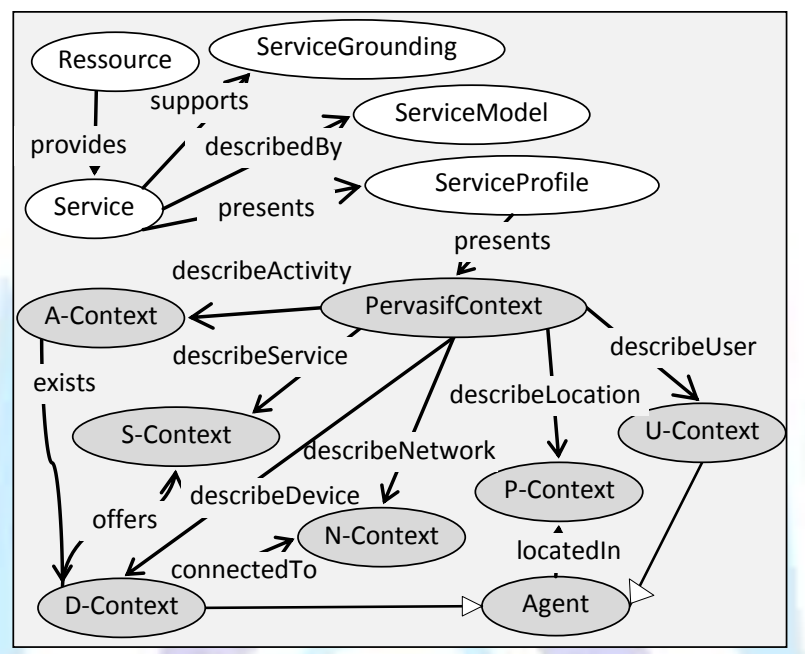

Fig. 5: Proposed structure for the first level of description

Each of the classes presented in the proposed OWL-S structure will be transformed in ontology. The latter regroups the classes and the attributes shown by the OWL semantic relation "owl:onProperty". The ontological structures are used to detail the contexts defined in the pervasive system.

\section{The second level of the web service description}

The second level is the most detailed contextual description. It is at this level that we discover the low-level description of the pervasive system (see Figure 4). To define each context, we have to get a set of extended OWL-S structures corresponding to the number of contexts described in a pervasive system.

\section{The OWL-S extension to describe the Physical Context (OWL-SPHC)}

The suggested OWL-S extension of the physical Context is an ontology that describes the user physical context as well as the set of devices and persons existent in a pervasive system. The physical context includes the contextual information related to the location and the location coordinates of an agent in a pervasive system. The idea here is to add the physical context description to the existing OWL-S ontological structure. The OWL-S extension structure is suggested in figure 6.

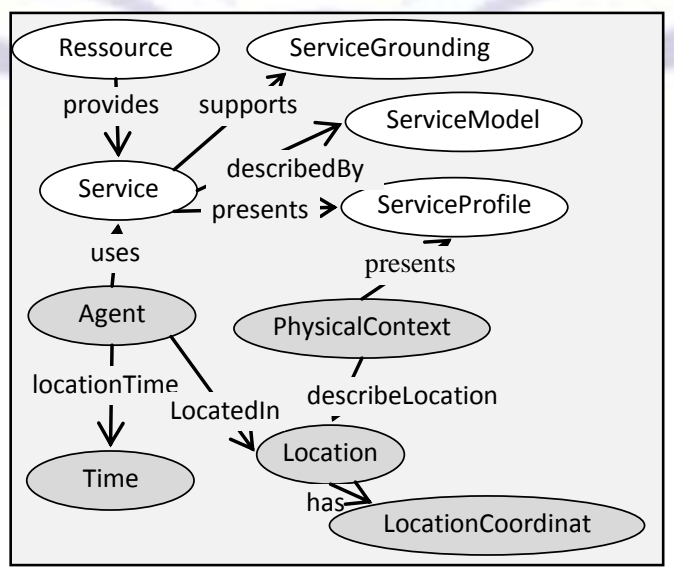

Fig. 6: The structure OWL-SPHC 
The adaptation of the user to the physical context is mainly manifested by the constraints expressed by the rules related to the execution of the applications in a pervasive system. These constraints can be summarized by the example in which an application is running in a location such as train station (where there is huge noise) or in a location such as a house (where there is silence). The time constraints occur when an application is executed in the same location but at different times. For instance, the execution constraints of an application during a work break are not the same as when it is running.

\section{The OWL-S extension to describe the Application Context (OWL-SAC)}

The OWL-S structure is designed to describe the application context is an ontology structure that defines the activity context executed in the pervasive system. The activity context includes both the contextual information related to the activity and the application that uses the rules in a pervasive system. The idea here is to add the activity context description to a standard OWL-S ontology. We present the suggested structure of the OWL-S extension in the following figure (see Figure 7).

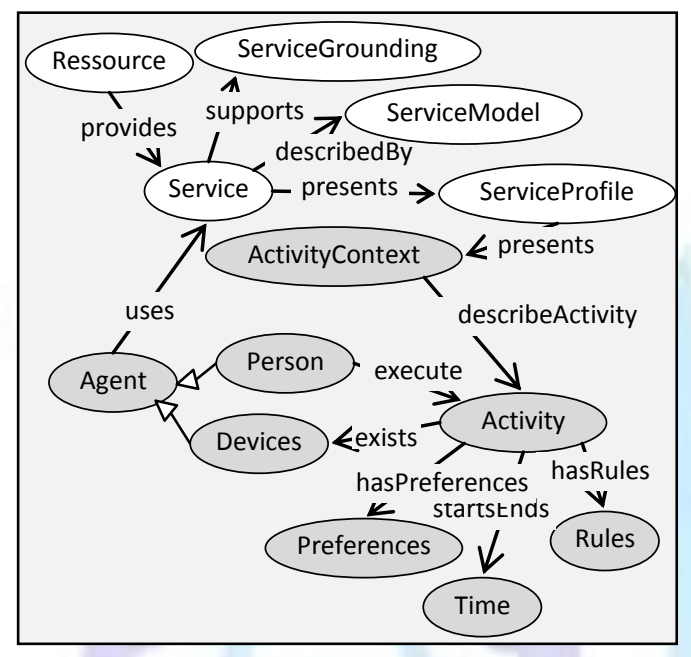

Fig. 7: The structure OWL-SAC

The adaptation of an activity in a pervasive system manifests itself in the activity characteristics and the rules implementing this activity. The devices characteristics may affect the activity performance. The activity execution begins and ends at a given moment in time. A computer activity can be executed in a specific platform may not be executed in another environment. In other words, audio or multimedia activity can be executed only in multimedia environments.

\section{The OWL-S extension to describe the User Context (OWL-SUC)}

In fact, the user is the focal point of any information system. In the pervasive system, the user is defined by two information characteristics: the static characteristics (name, age, sex, etc.) and the dynamic characteristics. The dynamic characteristics are defined by the preferences and the user's environment (location, time, etc.). To describe the user context, we consider the OWL-S extension structure presented in figure 8 which combines the two types of characteristics of the user context.

The adaptation of the applications to the user's characteristics manifests itself essentially during the interaction between the human-machine interfaces and the method of executing an activity. An activity can be executed according to the user's preferences. In addition, the user's static characteristics can impact on the methods of using a pervasive system since this type of system is adaptable to any user.

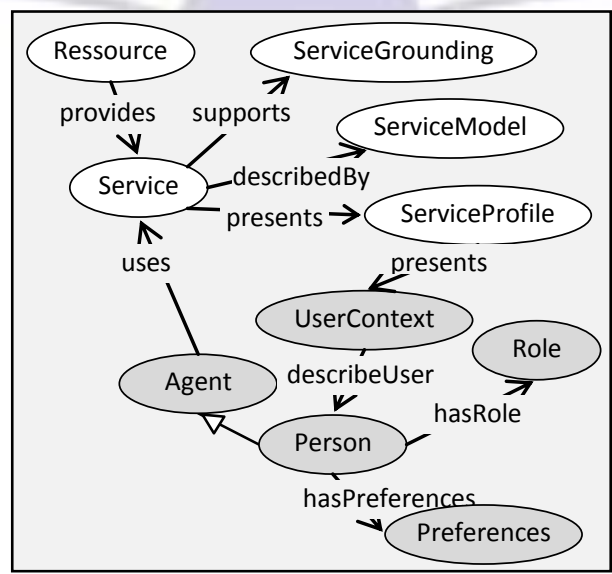

Fig. 8: The structure OWL-SUC 


\section{The OWL-S extension to describe the Service Context (OWL-SSC)}

The OWL-S structure used to describe the service context as an ontology which contains information about the services provided by the pervasive system. Each service in the pervasive system has a set of rules. Since we have an intelligent system, this system must provide services according to the user's desires and preferences such as the physical features, the available networks, etc. The OWL-S ontology is proposed as follows (see Figure 9).

This ontology is meant to describe the services provided by the pervasive system and to adapt an application to the user's desires and preferences. In other words, a single system must interact with any type of users, networks, devices, etc.

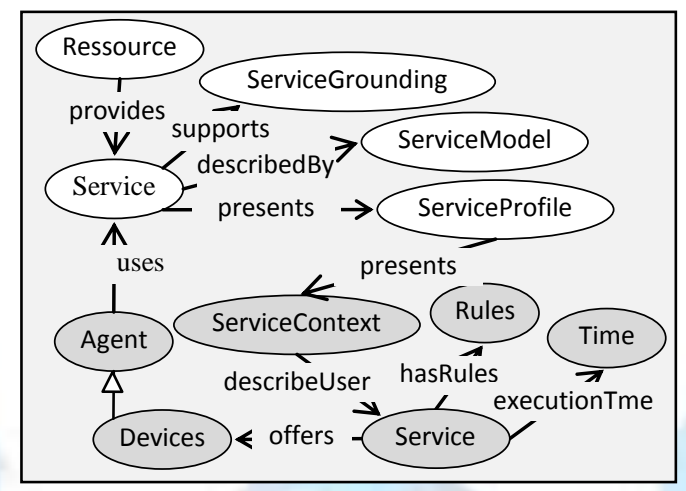

Fig. 9: The OWL-SSC structure

\section{The OWL-S extension to describe the Device Context (OWL-SDC)}

The OWL-S structure is created to describe the device context and the activity profiles in the pervasive information system. Each device has a configuration, rules and preferences. In the mobile system, a new communication method has emerged to satisfy the user's needs. Such a method paves the way for the propagation of intelligent systems through the invention of smart phones such as the Blackberrys, the iPhone... and touch pads such as the iPad. In order to ensure the adaptation, the pervasive system must capture a material list to ensure the answer to the query in accordance with the hardware configuration (see Figure 10).

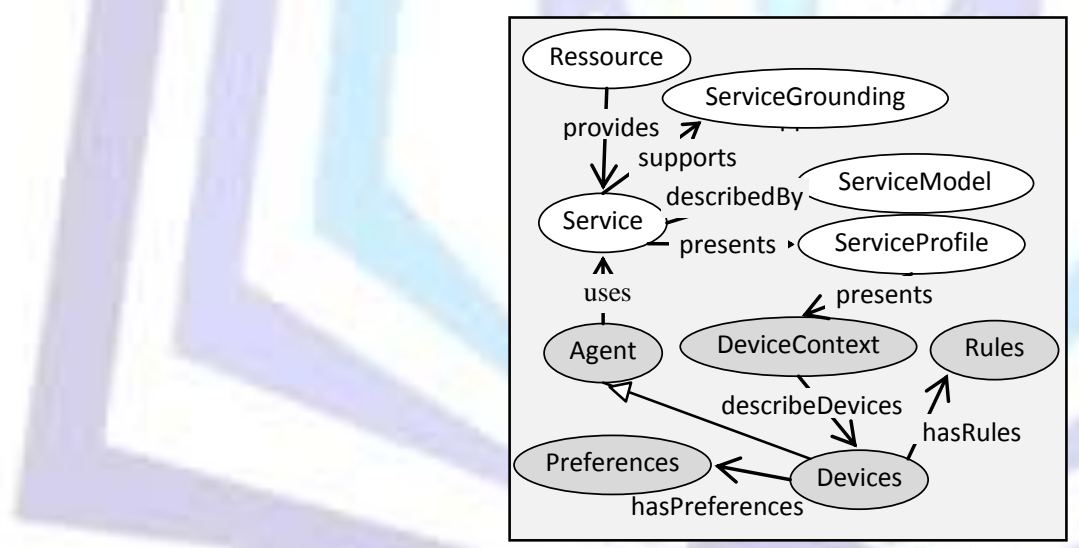

Fig. 10: The structure OWL-SDC

The material side is very important since the pervasive system is accessible anywhere, anyhow and to anything. Indeed, such a system can be executed according to the existing hardware.

\section{The OWL-S extension to describe the Network Context (OWL-SNC)}

The proposed OWL-S structure regroups the network context description and combines the characteristics of all the available networks in the pervasive information system. In this type of information system, there are several types of wireless networks (3G, WiFi, GSM, UMTS ...). Actually, each type of network has its own characteristics, access rules and preferences. To describe the network context, we propose the following OWL-S structure extension (see Figure 11).

The network selection or adaptation in the pervasive system is provided by the proposed ontology (see Figure 11) since the web service will use this structure to describe its services semantically. We can give real examples to describe the network in a pervasive system: the traveler can check and send his documents according to the characteristics of the wireless networks available in the airport, the patient can make an appointment through his PDA, the manager can supervise the workers remotely, etc. 


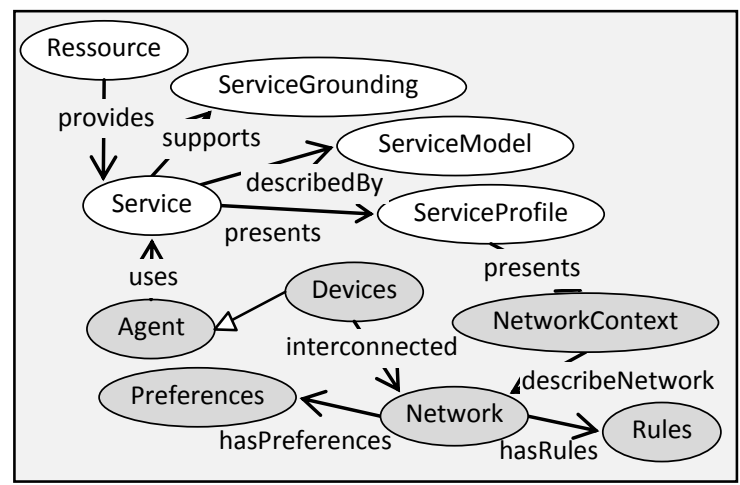

Fig. 11: The OWL-SNC structure

\section{An Example of using the proposed OWL-S extensions}

In this paper, we present an OWL-S extension for each context. In this section, we present a scenario to give an idea about the description related to the pervasive system.

The presented scenario (see Figure 11) is obtained after applying the representation of the rules exemplified in the figure below. In other words, the rules are defined by a set of conditions. The latter resulted from the user's request values by means of logic operators ("AND" or "OR"). The value used in this presentation is manipulated by the two operations "getValue()" and "setService()". The result may be one service or several services according to the logic operator.

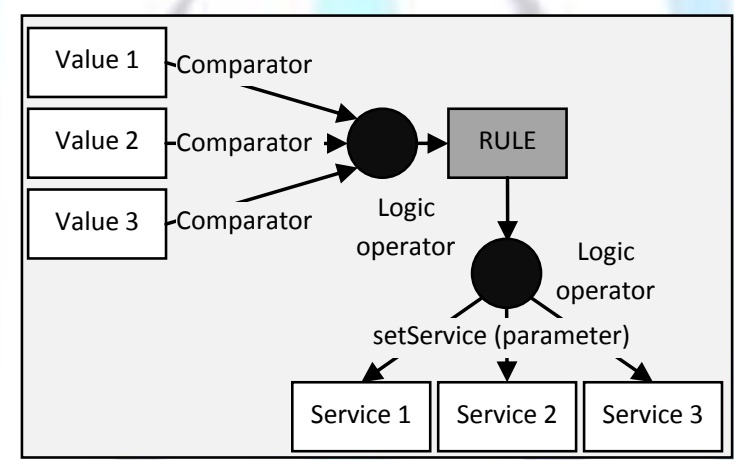

Fig. 12: The rule representation

The scenario definition: On a train, a passenger uses his laptop. The laptop battery level averages $50 \%$. Consequently, the volume of all multimedia document audio media should be set at $70 \%$ and the brightness level of the used device must be reduced to $50 \%$.

The scenario result: The scenario described in the scheme of figure 13 includes three contexts defined in a pervasive system: the physical context ("PhysicalContext"), the device context ("DeviceContext") and the application or activity context ("ActivityContext"). Each context provides information to have the system adapted to the user situation. In the physical context, the location characteristics are stored through the class "Location".

Among these characteristics, we can mention the attribute "LocationName". This allows you to define the location of an agent. In this situation, we notice that there are three possible values for this attribute (airport, train and house). In the device context, the class ("Device") includes all the features related to the material used in the pervasive system. Among the device class characteristics in this scenario we define three attributes: the battery level ("BatteryLevel"), the used equipment type ("DeviceType") and the brightness level ("BrightnessLevel").

Each of these attributes has values stored in the structure of the proposed context description. The attribute "DeviceType" has two values; PDA and portable. We note that there are three values to describe the brightness level ("BrightnessLevel"); " $50 \%$ ", " $30 \%$ " and "10\%" and for the battery level ("BatteryLevel"); " $50 \%$ ", " $70 \%$ " and "90\%". The activity context ("ActivityContext") in this situation is described by the two classes "Activity" and "rules". Each activity ("Activity") has its own rules ("rules").

The activity type is an attribute in the class ("ActivityType"). It includes, in this scenario, two activity types; "chat on facebook" and "audio clip player". The last type is selected by the system. To implement this activity, the volume of the audio sequence is one of the rules. It is secured by three possible values: " $30 \%$ ", " $50 \%$ " and " $70 \%$ ". 


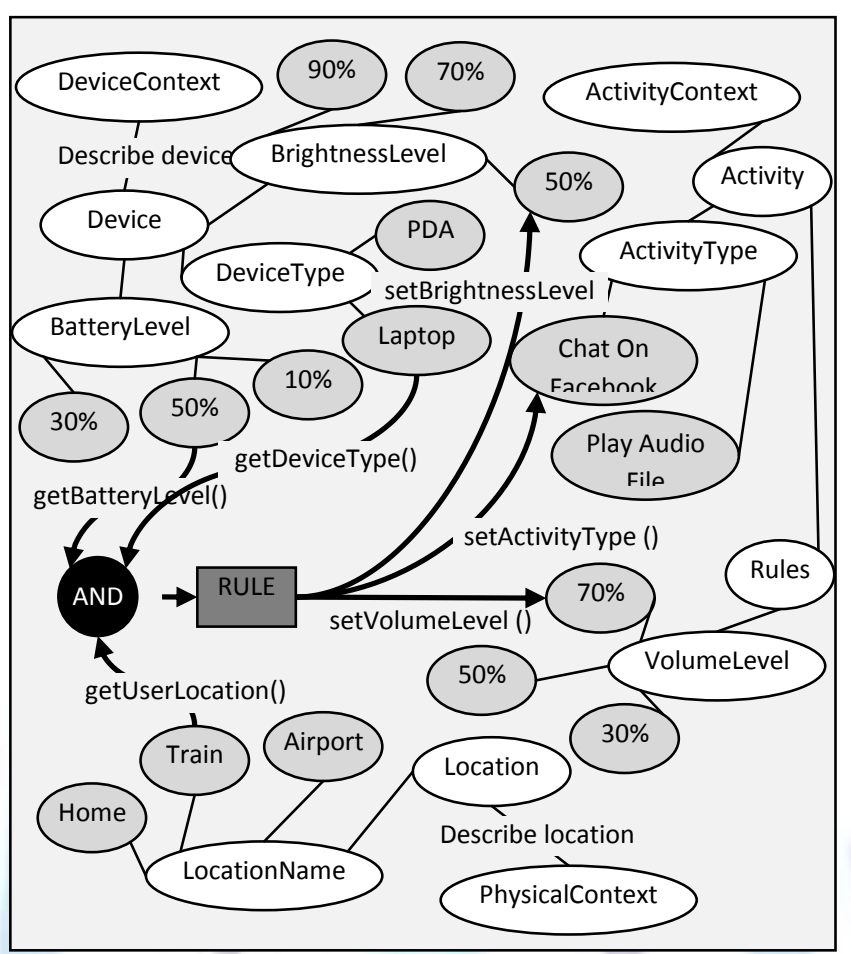

Fig. 13: The scenario application to the context

\section{CONCLUSION}

In this present paper, we present the contextual information related to the pervasive information system based on a generic model. Hence, we suggest a classification of this information. The result of this classification is six ontology; the user context ontology, the physical context ontology, the network context ontology, the activity context ontology, the device context ontology, and the service context ontology.

This set of ontology is integrated into an existing OWL-S structure to create the semantic web services and to determine the second level of description. Also, we propose a generic description for the first level. In future work, we intend to suggest a set of generic rules that can be applied to this ontology to adapt the functionality of the pervasive system.

\section{REFERENCES}

[1] A. K. Dey. Understanding and using context. Personal and Ubiquitous Computing, 5:4\{7, 2001.

[2] A. Fatma, J. Anis, and G. Faiez. The generic model for pervasive information system. In Proceedings of the 3rd International Conference on Information and Communication Systems, ICICS '12, pages 1--7, New York, NY, USA, 2012. ACM.

[3] D. Martin, M. Burstein, and J. H. et alt. Owl-s: Semantic markup for web services. Member submission, W3C, Nov. 2004. http://www.w3.org/Submission/OWL-S/.

[4] D. L. Martin, M. H. Burstein, D. V. McDermott, S. A. Mcllraith, M. Paolucci, K. P. Sycara, D. L. McGuinness, E. Sirin, and N. Srinivasan. Bringing semantics to web services with owl-s. In World Wide Web, pages 243--277, 2007.

[5] S. B. Mokhtar, D. Fournier, N. Georgantas, V. Issarny, S. B. Mokhtar, D. Fournier, , and NikolaosGeorgantas. Context-aware service composition in pervasive computing environments. In In RISE,volume3943ofLNCS,pages 129 144. Springer, 2005.

[6] I.-S. Park, W.-T. Kim, and Y.-J. Park. A ubiquitous streaming framework for multimedia broadcasting services with qos based mobility support. In H.-K. Kahng, editor, Information Networking, Networking Technologies for Broadband and Mobile Networks, International Conference ICOIN 2004, Busan, Korea, February 18-20, 2004, Revised Selected Papers, volume 3090 of Lecture Notes in Computer Science, pages 65--74. Springer, 2004.

[7] L. Qiu, L. Chang, F. Lin, and Z. Shi. Context optimization of ai planning for semantic web services composition. Service Oriented Computing and Applications, 1(2):117\{128, 2007.

[8] L. Qiu, Z. Shi, and F. Lin. Context optimization of ai planning for services composition. In ICEBE, pages 610--617, 2006.

[9] M. Weiser. The computer for the twenty-first century, Sept. 1991. 